\title{
Hilbert's Nullstellensatz and an Algorithm for Proving Combinatorial Infeasibility
}

\author{
J.A. De Loera* \\ Dept. of Mathematics \\ Univ. of California, Davis \\ Davis, CA \\ deloera@math.ucdavis.edu \\ P.N. Malkin \\ Dept. of Mathematics \\ Univ. of California, Davis \\ Davis, CA \\ malkin@math.ucdavis.edu
}

\author{
J. Lee \\ Mathematical Programming \\ IBM T.J. Watson Res. Cntr. \\ Yorktown Heights, New York \\ jonlee@us.ibm.com \\ S. Margulies \\ Dept. of Computer Science \\ Univ. of California, Davis \\ Davis, CA \\ smargulies@ucdavis.edu
}

\begin{abstract}
Systems of polynomial equations over an algebraically-closed field $\mathbb{K}$ can be used to concisely model many combinatorial problems. In this way, a combinatorial problem is feasible (e.g., a graph is 3-colorable, hamiltonian, etc.) if and only if a related system of polynomial equations has a solution over $\mathbb{K}$. In this paper, we investigate an algorithm aimed at proving combinatorial infeasibility based on the observed low degree of Hilbert's Nullstellensatz certificates for polynomial systems arising in combinatorics and on large-scale linear-algebra computations over $\mathbb{K}$. We report on experiments based on the problem of proving the non-3-colorability of graphs. We successfully solved graph problem instances having thousands of nodes and tens of thousands of edges.
\end{abstract}

\section{Categories and Subject Descriptors}

G.2.1 [Combinatorics]: Combinatorial algorithms

\section{General Terms}

Algorithms

\section{INTRODUCTION}

It is well known that systems of polynomial equations over a field can yield small models of difficult combinatorial problems. For example, it was first noted by D. Bayer that the 3-colorability of graphs can be modeled via a system of polynomial equations [2]. More generally, one can easily prove the following: *Research supported in part by an IBM Open Collaborative
Research Award and by NSF grant DMS-0608785

Permission to make digital or hard copies of all or part of this work for personal or classroom use is granted without fee provided that copies are not made or distributed for profit or commercial advantage and that copies bear this notice and the full citation on the first page. To copy otherwise, to republish, to post on servers or to redistribute to lists, requires prior specific permission and/or a fee.

ISSAC'08, July 20-23, 2008, Hagenberg, Austria.

Copyright 2008 ACM 978-1-59593-904-3/08/07 ...\$5.00.
LemMa 1.1. A graph $G$ is $k$-colorable iff the system of $n+m$ equations in $n$ variables $x_{i}^{k}-1=0, \forall i \in V(G)$, and $\sum_{l=0}^{k-1} x_{i}^{k-1-l} x_{j}^{l}=0, \forall\{i, j\} \in E(G)$ has a complex solution. Moreover, the number of solutions equals the number of distinct $k$-colorings multiplied by $k$ !.

Although such polynomial system encodings have been used to prove combinatorial results (see $[1,6]$ and therein), they have not been widely used for computation. The key issue that we investigate here is the use of such polynomial systems to effectively decide whether a graph, or other combinatorial structure, has a property captured by the polynomial system and its associated ideal. We call this the combinatorial feasibility problem. We are particularly interested in whether this can be accomplished in practice for large combinatorial structures such as graphs with many nodes.

Certainly, using standard tools in computational algebra such as Gröbner bases, one can answer the combinatorial feasibility problem by simply solving the system of polynomials. Nevertheless, it has been shown by experiments that current Gröbner bases implementations often cannot directly solve polynomial systems with hundreds of polynomials. This paper proposes another approach that relies instead on the nice low degree of the Hilbert's Nullstellensatz for combinatorial polynomial systems and on large-scale linear-algebra computation.

For a hard combinatorial problem (e.g., 3-colorability of graphs), we associate a system of polynomial equations $J=$ $\left\{f_{1}(x)=0, \ldots, f_{s}(x)=0\right\}$ such that the system $J$ has a solution if and only if the combinatorial problem has a feasible solution. The Hilbert Nullstellensatz (see e.g.,[5]) states that the system of polynomial equations has no solution over an algebraically-closed field $\mathbb{K}$ if and only if there exist polynomials $\beta_{1}, \ldots, \beta_{s} \in \mathbb{K}\left[x_{1}, \ldots, x_{n}\right]$ such that $1=\sum \beta_{i} f_{i}$. Thus, if the polynomial system $J$ has no solution, then there exists a certificate that $J$ has no solution, and thus a certificate that the combinatorial problem is infeasible.

The key idea that we explore in this article is to use the Nullstellensatz to generate a finite sequence of linear algebra systems, of increasing size, which will eventually become feasible if and only if the combinatorial problem is infeasible. Given a system of polynomial equations, we fix a tentative degree $k$ for the coefficient polynomials $\beta_{i}$ in the certificates. 
We can decide whether there is a Nullstellensatz certificate with coefficients of degree $\leq k$ by solving a system of linear equations over the field $\mathbb{K}$ whose variables are in bijection with the coefficients of the monomials of the polynomials $\beta_{1}, \ldots, \beta_{s}$. If this linear system has a solution, we have found a certificate; otherwise, we try a higher degree for the polynomials $\beta_{i}$. This process is guaranteed to terminate because, for a Nullstellensatz certificate to exist, the degrees of the polynomials $\beta_{i}$ cannot be more than known bounds (see [10] and references therein). We explain the details of the algorithm, which we call NulLA, in Section 2.

Our method can be seen as a general-field variation of work by Lasserre [11], Laurent [13] and Parrilo [16] and many others, who studied the problem of minimizing a general polynomial function $f(x)$ over a real algebraic variety with finitely many points. Laurent proved that when the variety consists of the solutions of a zero-dimensional radical ideal $I$, one can set up the optimization problem $\min \{f(x): x \in \operatorname{variety}(I)\}$ as a finite sequence of semidefinite programs terminating with the optimal solution (see [13]). There are two key observations that speed up practical calculations considerably: (1) when dealing with feasibility, instead of optimization, linear algebra replaces semidefinite programming and (2) there are ways of controlling the length of the sequence of linear-algebra systems including finite field computation instead of calculations over the reals and the reduction of matrix size by symmetries. See Section 3 for details.

Our algorithm has good practical performance and numerical stability. Although known theoretical bounds for degrees of the Nullstellensatz coefficients are doubly-exponential in the size of the polynomial system (and indeed there exist examples that attain such a large bound and make NulLA useless in general), our experiments demonstrate that often low degrees suffice for systems of polynomials coming from graphs. We have implemented an exact-arithmetic linear system solver optimized for these Nullstellensatz-based systems. We performed many experiments using NulLA, focusing on the problem of deciding graph 3-colorability (note that the method is applicable to any combinatorial problem whose polynomial system encoding is known). We conclude with a report on these experiments in Section 4.

\section{NULLSTELLENSATZ LINEAR ALGEBRA (NULLA) ALGORITHM}

Recall that Hilbert's Nullstellensatz states that a system of polynomial equations $f_{1}(x)=0, \ldots, f_{s}(x)=0$, where $f_{i} \in \mathbb{K}\left[x_{1}, \ldots, x_{n}\right]$ and $\mathbb{K}$ is an algebraically closed field, has no solution in $\mathbb{K}^{n}$ if and only if there exist polynomials $\beta_{1}, \ldots, \beta_{s} \in \mathbb{K}\left[x_{1}, \ldots, x_{n}\right]$ such that $1=\sum \beta_{i} f_{i}[5]$. The polynomial identity $1=\sum \beta_{i} f_{i}$ is called a Nullstellensatz certificate, which has degree $d$ if $\max \left\{\operatorname{deg}\left(\beta_{i}\right)\right\}=d$.

The Nullstellensatz Linear Algebra (NulLA) algorithm accepts as input a system of polynomial equations and outputs either a yes answer, if the system of polynomial equations has a solution, or a no answer, along with a Nullstellensatz infeasibility certificate, if the system has no solution. Before stating the algorithm in pseudocode, we clarify the connection to linear algebra. Suppose the input polynomial system is infeasible over $\mathbb{K}$, and suppose further that an oracle has told us the certificate has degree $d$ but that we do not know the actual coefficients of the degree $d$ polynomials $\beta_{i}$. Thus, we have the polynomial identity $1=\sum \beta_{i} f_{i}$. If we expand the identity into monomials, the coefficients of a monomial are linear expressions in the coefficients of the $\beta_{i}$. Since two polynomials over a field are identical precisely when the coefficients of corresponding monomials are identical, from the $1=\sum \beta_{i} f_{i}$, we get a system of linear equations whose variables are the coefficients of the $\beta_{i}$. Here is an example:

EXAMPLE 2.1. Consider the polynomial system $x_{1}^{2}-1=$ $0, x_{1}+x_{2}=0, x_{1}+x_{3}=0, x_{2}+x_{3}=0$. This system has no solution, and a Nullstellensatz certificate of degree one.

$$
\begin{aligned}
1= & \underbrace{\left(c_{0} x_{1}+c_{1} x_{2}+c_{2} x_{3}+c_{3}\right)}_{\beta_{1}} \underbrace{\left(x_{1}^{2}-1\right)}_{\beta_{2}} \\
& +\underbrace{\left(c_{4} x_{1}+c_{5} x_{2}+c_{6} x_{3}+c_{7}\right)}_{\beta_{3}} \underbrace{\left(x_{1}+x_{2}\right)}_{f_{2}} \\
& +\underbrace{\left(c_{8} x_{1}+c_{9} x_{2}+c_{10} x_{3}+c_{11}\right)}_{\beta_{4}} \underbrace{\left(x_{1}+x_{3}\right)}_{f_{3}} \\
& +\underbrace{\left(c_{12} x_{1}+c_{13} x_{2}+c_{14} x_{3}+c_{15}\right)}_{f_{12}} \underbrace{\left(x_{2}+x_{3}\right)}_{f_{4}} .
\end{aligned}
$$

Expanding the tentative Nullstellensatz certificate into monomials and grouping like terms, we arrive at the following polynomial equation:

$$
\begin{aligned}
1 & =c_{0} x_{1}^{3}+c_{1} x_{1}^{2} x_{2}+c_{2} x_{1}^{2} x_{3}+\left(c_{3}+c_{4}+c_{8}\right) x_{1}^{2}-c_{3} \\
& +\left(c_{10}+c_{14}\right) x_{3}^{2}+\left(c_{4}+c_{5}+c_{9}+c_{12}\right) x_{1} x_{2}+\left(c_{5}+c_{13}\right) x_{2}^{2} \\
& +\left(c_{6}+c_{8}+c_{10}+c_{12}\right) x_{1} x_{3}+\left(c_{6}+c_{9}+c_{13}+c_{14}\right) x_{2} x_{3} \\
& +\left(c_{7}+c_{15}-c_{1}\right) x_{2}+\left(c_{11}+c_{15}-c_{2}\right) x_{3}+\left(c_{7}+c_{11}-c_{0}\right) x_{1} .
\end{aligned}
$$

From this, we extract a system of linear equations. Since a Nullstellensatz certificate is identically one, all monomials except the constant term must be equal to zero; namely:

$$
c_{0}=0, \quad c_{11}+c_{15}-c_{2}=0, \quad-c_{3}=1 .
$$

By solving the system of linear equations, we reconstruct the Nullstellensatz certificate from the solution. Indeed

$$
1=\frac{1}{2} x_{1}\left(x_{1}+x_{2}\right)-\frac{1}{2} x_{1}\left(x_{2}+x_{3}\right)+\frac{1}{2} x_{1}\left(x_{1}+x_{3}\right)-\left(x_{1}^{2}-1\right)
$$

In general, one does not know the degree of the Nullstellensatz certificate in advance. What one can do is to start with a tentative degree, say start at degree one, produce the corresponding linear system, and solve it. If the system has a solution, then we have found a Nullstellensatz certificate demonstrating that the original input polynomials do not have a common root. Otherwise, we increment the degree until we can be sure that there will not be a Nullstellensatz certificate at all, and thus we can conclude the system of polynomials has a solution. The number of iterations of the above steps determines the running time of NulLA. For this, there are well-known upper bounds on the degree of the Nullstellensatz certificate [10]. These upper bounds for the degrees of the coefficients $\beta_{i}$ in the Hilbert Nullstellensatz certificates for general systems of polynomials are doublyexponential in the number of input polynomials and their degree. Unfortunately, these bounds are known to be sharp for some specially-constructed systems. Although this immediately says that NulLA is not practical for arbitrary polynomial systems, we have observed in practice that polynomial systems for combinatorial questions are extremely specialized, and the degree growth is often very slow - enough to deal with large graphs or other combinatorial structures. Now we describe NulLA in pseudocode: 


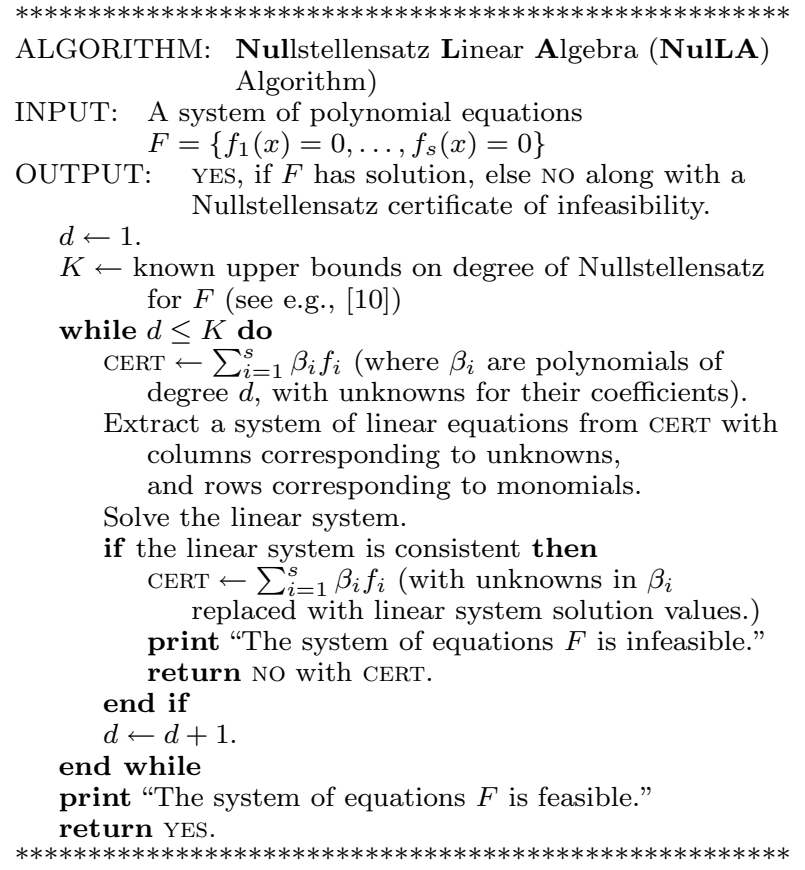

This opens several theoretical questions. It is natural to ask about lower bounds on the degree of the Nullstellensatz certificates. Little is known, but recently it was shown in [6], that for the problem of deciding whether a given graph $G$ has an independent set of a given size, a minimum-degree Nullstellensatz certificate for the non-existence of an independent set of size greater than $\alpha(G)$ (the size of the largest independent set in $G$ ) has degree equal to $\alpha(G)$, and it is very dense; specifically, it contains at least one term per independent set in $G$. For polynomial systems coming from logic there has also been an effort to show degree growth in related polynomial systems (see $[3,8]$ and the references therein). Another question is to provide tighter, more realistic upper bounds for concrete systems of polynomials. It is a challenge to settle it for any concrete family of polynomial systems.

\section{FOUR MATHEMATICAL IDEAS TO OP- TIMIZE NULLA}

Since we are interested in practical computational problems, it makes sense to explore refinements and variations that make NulLA robust and much faster for concrete challenges. The main computational component of NulLA is to construct and solve linear systems for finding Nullstellensatz certificates of increasing degree. These linear systems are typically very large for reasonably-sized problems, even for certificate degrees as low as four, which can produce linear systems with millions of variables (see Section 4). Furthermore, the size of the linear system increases dramatically with the degree of the certificate. In particular, the number of variables in the linear system to find a Nullstellensatz certificate of degree $d$ is precisely $s\left(\begin{array}{c}n+d \\ d\end{array}\right)$ where $n$ is the number of variables in the polynomial system and $s$ is the number of polynomials. Note that $\left(\begin{array}{c}n+d \\ d\end{array}\right)$ is the number of possible monomials of degree $d$ or less. Also, the number of non-zero entries in the constraint matrix is precisely $M\left(\begin{array}{c}n+d \\ d\end{array}\right)$ where $M$ is the sum over the number of monomials in each polynomial of the system.
For this reason, in this section, we explore mathematical approaches for solving the linear system more efficiently and robustly, for decreasing the size of the linear system for a given degree, and for decreasing the degree of the Nullstellensatz certificate for infeasible polynomial systems thus significantly reducing the size of the largest linear system that we need to solve to prove infeasibility. Note that these approaches to reduce the degree do not necessarily decrease the available upper bound on the degree of the Nullstellensatz certificate required for proving feasibility.

It is certainly possible to significantly decrease the size of the linear system by preprocessing the given polynomial system to remove redundant polynomial equations and also by preprocessing the linear system itself to eliminate many variables. For example, in the case of 3-coloring problems for connected graphs, since $\left(x_{i}^{3}+1\right)=\left(x_{j}^{3}+1\right)+\left(x_{i}+\right.$ $\left.x_{j}\right)\left(x_{i}^{2}+x_{i} x_{j}+x_{j}^{2}\right)$, we can remove all but one of the vertex polynomials by tracing paths through the graph. However, preprocessing alone is not sufficient to enable us to solve some large polynomial systems.

The mathematical ideas we explain in the rest of this section can be applied to arbitrary polynomial systems, but to implement them, one has to look for the right structures in the polynomials.

\subsection{NulLA over Finite Fields}

The first idea is that, for combinatorial problems, one can often carry out calculations over finite fields instead of relying on unstable floating-point calculations. We illustrate this with the problem of deciding whether the vertices of a graph permit a proper 3-coloring. The following encoding (a variation of [2] over the complex numbers) allows us to compute over $\mathbb{F}_{2}$, which is robust and much faster in practice (also see [7]):

LEMMA 3.1. The graph $G$ is 3-colorable if and only if the zero-dimensional system of equations $x_{i}^{3}+1=0, \forall i \in V(G)$, and $x_{i}^{2}+x_{i} x_{j}+x_{j}^{2}=0, \forall\{i, j\} \in E(G)$, has a solution over $\overline{\mathbb{F}}_{2}$, the algebraic closure of $\mathbb{F}_{2}$.

Before we prove Lemma 3.1, we introduce a convenient notation: Let $\alpha$ be an algebraic element over $\overline{\mathbb{F}}_{2}$ such that $\alpha^{2}+\alpha+1=0$. Thus, although $x_{i}^{3}+1$ has only one root over $\mathbb{F}_{2}$, since $x_{i}^{3}+1=\left(x_{i}+1\right)\left(x_{i}^{2}+x_{i}+1\right)$, the polynomial $x_{i}^{3}+1$ has three roots over $\overline{\mathbb{F}}_{2}$, which are $1, \alpha$ and $\alpha+1$.

Proof. If the graph $G$ is 3 -colorable, simply map the three colors to $1, \alpha$ and $\alpha+1$. Clearly, the vertex polynomial equations $x_{i}^{3}+1=0$ are satisfied. Furthermore, given an edge $\{i, j\}, x_{i}+x_{j} \neq 0$ since variable assignments correspond to a proper 3 -coloring and adjacent vertices are assigned different roots. This implies that $x_{i}^{3}+x_{j}^{3}=\left(x_{i}+x_{j}\right)\left(x_{i}^{2}+\right.$ $\left.x_{i} x_{j}+x_{j}^{2}\right)=1+1=0$. Therefore, $x_{i}^{2}+x_{i} x_{j}+x_{j}^{2}=0$ and the edge polynomial equations are satisfied.

Conversely, suppose that there exists a solution to the system of polynomial equations. Clearly, every vertex is assigned either $1, \alpha$ or $\alpha+1$. We will show that adjacent vertices are assigned different values. Our proof is by contradiction: Assume that two adjacent vertices $i, j$ are assigned the same value $\beta$. Then, $0=x_{i}^{2}+x_{i} x_{j}+x_{j}^{2}=\beta^{2}+\beta^{2}+\beta^{2}=$ $3 \beta^{2} \neq 0$. Therefore, adjacent vertices are assigned different roots, and a solution to the system corresponds directly to a proper 3-coloring. 
We remark that this result can be extended to $k$-colorability and $\overline{\mathbb{F}}_{q}$, when $q$ is relatively prime to $k$. The following computational lemma will allow us to certify graph non-3colorability very rapidly over $\mathbb{F}_{2}$ instead of working over its algebraic closure.

Lemma 3.2. Let $\mathbb{K}$ be a field and $\overline{\mathbb{K}}$ its algebraic closure. Given $f_{1}, f_{2}, \ldots, f_{s} \in \mathbb{K}\left[x_{1}, \ldots, x_{n}\right]$, there exists a Nullstellensatz certificate $1=\sum \beta_{i} f_{i}$ where $\beta_{i} \in \overline{\mathbb{K}}\left[x_{1}, \ldots, x_{n}\right]$ if and only if there exists a Nullstellensatz certificate $1=\sum \beta_{i}^{\prime} f_{i}$ where $\beta_{i}^{\prime} \in \mathbb{K}\left[x_{1}, \ldots, x_{n}\right]$.

Proof. If there exists a Nullstellensatz certificate $1=$ $\sum \beta_{i} f_{i}$ where $\beta_{i} \in \overline{\mathbb{K}}\left[x_{1}, \ldots, x_{n}\right]$, via NulLA, construct the associated linear system and solve. Since $f_{i} \in \mathbb{K}\left[x_{1}, \ldots, x_{n}\right]$, the coefficients in the linear system will consist only of values in $\mathbb{K}$. Thus, solving the linear system relies only on computations in $\mathbb{K}$, and if the free variables are chosen from $\mathbb{K}$ instead of $\overline{\mathbb{K}}$, the resulting Nullstellensatz certificate $1=\sum \beta_{i}^{\prime} f_{i}$ has $\beta_{i}^{\prime} \in \mathbb{K}\left[x_{1}, \ldots, x_{n}\right]$. The reverse implication is trivial. $\square$

Therefore, we have the following corollary:

Corollary 3.3. A graph $G$ is non-3-colorable if and only if there exists a Nullstellensatz certificate $1=\sum \beta_{i} f_{i}$ where $\beta_{i} \in \mathbb{F}_{2}\left[x_{1}, \ldots, x_{n}\right]$ where the polynomials $f_{i} \in \mathbb{F}_{2}\left[x_{1}, \ldots, x_{n}\right]$ are as defined in Lemma 3.1.

This corollary enables us to compute over $\mathbb{F}_{2}$, which is extremely fast in practice (see Section 4).

Finally, the degree of Nullstellensatz certificates necessary to prove infeasibility can be lower over $\mathbb{F}_{2}$ than over the rationals. For example, over the rationals, every odd-wheel has a minimum non-3-colorability certificate of degree four [6]. However, over $\mathbb{F}_{2}$, every odd-wheel has a Nullstellensatz certificate of degree one. Therefore, not only are the mathematical computations more efficient over $\mathbb{F}_{2}$ as compared to the rationals, but the algebraic properties of the certificates themselves are sometimes more favorable for computation as well.

\subsection{NulLA with symmetries}

Let us assume that the input polynomial system $F=$ $\left\{f_{1}, \ldots, f_{s}\right\}$ has maximum degree $q$ and that $n$ is the number of variables present. As we observed in Section 2, for a given fixed positive integer $d$ serving as a tentative degree for the Nullstellensatz certificate, the Nullstellensatz coefficients come from the solution of a system of linear equations. We now take a closer look at the matrix equation $M_{F, d} y=b_{F, d}$ defining the system of linear equations. First of all, the matrix $M_{F, d}$ has one row per monomial $x^{\alpha}$ of degree less than or equal to $q+d$ on the $n$ variables and one column per polynomial of the form $x^{\delta} f_{i}$, i.e., the product of a monomial $x^{\delta}$ of degree less than or equal to $d$ and a polynomial $f_{i} \in$ $F$. Thus, $M_{F, d}=\left(M_{x^{\alpha}, x^{\delta} f_{i}}\right)$ where $M_{x^{\alpha}, x^{\delta} f_{i}}$ equals the coefficient of the monomial $x^{\alpha}$ in the polynomial $x^{\delta} f_{i}$. The variable $y$ has one entry for every polynomial of the form $x^{\delta} f_{i}$ denoted $y_{x^{\delta} f_{i}}$, and the vector $b_{F, d}$ has one entry for every monomial $x^{\alpha}$ of degree less than or equal to $q+d$ where $\left(b_{F, d}\right)_{x^{\alpha}}=0$ if $\alpha \neq 0$ and $\left(b_{F, d}\right)_{1}=1$.

Example 3.4. Consider the complete graph $K_{4}$. The shape of a degree-one Hilbert Nullstellensatz certificate over $\overline{\mathbb{F}}_{2}$ for non-3-colorability is as follows:

$$
1=c_{0}\left(x_{1}^{3}+1\right)
$$

$$
\begin{aligned}
& +\left(c_{12}^{1} x_{1}+c_{12}^{2} x_{2}+c_{12}^{3} x_{3}+c_{12}^{4} x_{4}\right)\left(x_{1}^{2}+x_{1} x_{2}+x_{2}^{2}\right) \\
& +\left(c_{13}^{1} x_{1}+c_{13}^{2} x_{2}+c_{13}^{3} x_{3}+c_{13}^{4} x_{4}\right)\left(x_{1}^{2}+x_{1} x_{3}+x_{3}^{2}\right) \\
& +\left(c_{14}^{1} x_{1}+c_{14}^{2} x_{2}+c_{14}^{3} x_{3}+c_{14}^{4} x_{4}\right)\left(x_{1}^{2}+x_{1} x_{4}+x_{4}^{2}\right) \\
& +\left(c_{23}^{1} x_{1}+c_{23}^{2} x_{2}+c_{23}^{3} x_{3}+c_{23}^{4} x_{4}\right)\left(x_{2}^{2}+x_{2} x_{3}+x_{3}^{2}\right) \\
& +\left(c_{24}^{1} x_{1}+c_{24}^{2} x_{2}+c_{24}^{3} x_{3}+c_{24}^{4} x_{4}\right)\left(x_{2}^{2}+x_{2} x_{4}+x_{4}^{2}\right) \\
& +\left(c_{34}^{1} x_{1}+c_{34}^{2} x_{2}+c_{34}^{3} x_{3}+c_{34}^{4} x_{4}\right)\left(x_{3}^{2}+x_{3} x_{4}+x_{4}^{2}\right)
\end{aligned}
$$

Note that we have preprocessed the certificate by removing the redundant polynomials $x_{i}^{3}+1$ where $i \neq 1$ and removing some variables that we know a priori can be set to zero, which results in a matrix with less columns. As we explained in Section 2, this certificate gives a linear system of equations in the variables $c_{0}$ and $c_{i j}^{k}$ (note that $k$ is a superscript and not an exponent). This linear system can be captured as the matrix equation $M_{F, 1} c=b_{F, 1}$ where the matrix $M_{F, 1}$ is as follows.

\begin{tabular}{|r||c|c|ccc|cccc|c|cccc|}
\hline & $c_{0}$ & $c_{12}^{1} c_{12}^{2} c_{12}^{3}$ & $c_{12}^{4}$ & $c_{13}^{1}$ & $c_{13}^{2}$ & $c_{13}^{3}$ & $c_{13}^{4}$ & $\cdots$ & $c_{34}^{1}$ & $c_{34}^{2}$ & $c_{34}^{3}$ & $c_{34}^{4}$ \\
\hline \hline 1 & 1 & 0 & 0 & 0 & 0 & 0 & 0 & 0 & 0 & $\cdots$ & 0 & 0 & 0 & 0 \\
$x_{1}^{3}$ & 1 & 1 & 0 & 0 & 0 & 1 & 0 & 0 & 0 & $\cdots$ & 0 & 0 & 0 & 0 \\
$x_{1}^{2} x_{2}$ & 0 & 1 & 1 & 0 & 0 & 0 & 1 & 0 & 0 & $\cdots$ & 0 & 0 & 0 & 0 \\
$x_{1}^{2} x_{3}$ & 0 & 0 & 0 & 1 & 0 & 1 & 0 & 1 & 0 &. & 0 & 0 & 0 & 0 \\
$x_{1}^{2} x_{4}$ & 0 & 0 & 0 & 0 & 1 & 0 & 0 & 0 & 1 & $\cdots$ & 0 & 0 & 0 & 0 \\
$x_{1} x_{2}^{2}$ & 0 & 1 & 1 & 0 & 0 & 0 & 0 & 0 & 0 & $\cdots$ & 0 & 0 & 0 & 0 \\
$x_{1} x_{2} x_{3}$ & 0 & 0 & 0 & 1 & 0 & 0 & 1 & 0 & 0 & $\cdots$ & 0 & 0 & 0 & 0 \\
$x_{1} x_{2} x_{4}$ & 0 & 0 & 0 & 0 & 1 & 0 & 0 & 0 & 0 & $\cdots$ & 0 & 0 & 0 & 0 \\
$x_{1} x_{3}^{2}$ & 0 & 0 & 0 & 0 & 0 & 1 & 0 & 1 & 0 & $\cdots$ & 1 & 0 & 0 & 0 \\
$x_{1} x_{3} x_{4}$ & 0 & 0 & 0 & 0 & 0 & 0 & 0 & 0 & 1 & $\cdots$ & 1 & 0 & 0 & 0 \\
$x_{1} x_{4}^{2}$ & 0 & 0 & 0 & 0 & 0 & 0 & 0 & 0 & 0 & $\cdots$ & 1 & 0 & 0 & 0 \\
$x_{2}^{3}$ & 0 & 0 & 1 & 0 & 0 & 0 & 0 & 0 & 0 & $\cdots$ & 0 & 0 & 0 & 0 \\
$x_{2}^{2} x_{3}$ & 0 & 0 & 0 & 1 & 0 & 0 & 0 & 0 & 0 & $\cdots$ & 0 & 0 & 0 & 0 \\
$x_{2}^{2} x_{4}$ & 0 & 0 & 0 & 0 & 1 & 0 & 0 & 0 & 0 & $\cdots$ & 0 & 0 & 0 & 0 \\
$x_{2} x_{3}^{2}$ & 0 & 0 & 0 & 0 & 0 & 0 & 1 & 0 & 0 & $\cdots$ & 0 & 1 & 0 & 0 \\
$x_{2} x_{3} x_{4}$ & 0 & 0 & 0 & 0 & 0 & 0 & 0 & 0 & 0 & $\cdots$ & 0 & 1 & 0 & 0 \\
$x_{2} x_{4}^{2}$ & 0 & 0 & 0 & 0 & 0 & 0 & 0 & 0 & 0 & $\cdots$ & 0 & 1 & 0 & 0 \\
$x_{3}^{3}$ & 0 & 0 & 0 & 0 & 0 & 0 & 0 & 1 & 0 & $\cdots$ & 0 & 0 & 1 & 0 \\
$x_{3}^{2} x_{4}$ & 0 & 0 & 0 & 0 & 0 & 0 & 0 & 0 & 1 & $\cdots$ & 0 & 0 & 1 & 1 \\
$x_{3} x_{4}^{2}$ & 0 & 0 & 0 & 0 & 0 & 0 & 0 & 0 & 0 & $\cdots$ & 0 & 0 & 1 & 1 \\
$x_{4}^{3}$ & 0 & 0 & 0 & 0 & 0 & 0 & 0 & 0 & 0 & $\cdots$ & 0 & 0 & 0 & 1 \\
\hline
\end{tabular}

Certainly the matrix $M_{F, d}$ is rather large already for small systems of polynomials. The main point of this section is to demonstrate how to reduce the size of the matrix by using a group action on the variables, e.g., using symmetries or automorphisms in a graph. Suppose we have a finite permutation group $G$ acting on the variables $x_{1}, \ldots, x_{n}$. Clearly $G$ induces an action on the set of monomials with variables $x_{1}, x_{2}, \ldots, x_{n}$ of degree $t$. We will assume that the set $F$ of polynomials is invariant under the action of $G$, i.e., $g\left(f_{i}\right) \in F$ for each $f_{i} \in F$. Denote by $x^{\delta}$, the monomial $x_{1}^{\delta_{1}} x_{2}^{\delta_{2}} \ldots x_{n}^{\delta_{n}}$, a monomial of degree $\delta_{1}+\delta_{2}+\cdots+\delta_{n}$. Denote by $\operatorname{Orb}\left(x^{\alpha}\right), \operatorname{Orb}\left(x^{\delta} f_{i}\right)$ the orbit under $G$ of monomial $x^{\alpha}$ and, respectively, the orbit of the polynomial obtained as the product of the monomial $x^{\delta}$ and the polynomial $f_{i} \in F$.

We now introduce a new matrix equation $\bar{M}_{F, d, G} \bar{y}=$ $\bar{b}_{F, d, G}$. The rows of the matrix $\bar{M}_{F, d, G}$ are indexed by the orbits of monomials $\operatorname{Orb}\left(x^{\alpha}\right)$ where $x^{\alpha}$ is a monomial of degree less than or equal to $q+d$, and the columns of $\bar{M}_{F, d, G}$ are indexed by the orbits of polynomials $\operatorname{Orb}\left(x^{\delta} f_{i}\right)$ where $f_{i} \in F$ and the degree of the monomial $x^{\delta}$ less than or equal to $d$. Then, let $\bar{M}_{F, d, G}=\left(\bar{M}_{O r b\left(x^{\alpha}\right), O r b\left(x^{\delta} f_{i}\right)}\right)$ where

$$
\bar{M}_{O r b\left(x^{\alpha}\right), \operatorname{Orb}\left(x^{\delta} f_{i}\right)}=\sum_{x^{\gamma} f_{j} \in \operatorname{Orb}\left(x^{\delta} f_{i}\right)} M_{x^{\alpha}, x^{\gamma} f_{j}} .
$$


Note that $M_{x^{\alpha}, x^{\delta} f_{i}}=M_{g\left(x^{\alpha}\right), g\left(x^{\delta} f_{i}\right)}$ for all $g \in G$ meaning that the coefficient of the monomial $x^{\alpha}$ in the polynomial $x^{\delta} f_{i}$ is the same as the coefficient of the monomial $g\left(x^{\alpha}\right)$ in the polynomial $g\left(x^{\delta} f_{i}\right)$. So, $\forall x^{d} \in \operatorname{Orb}\left(x^{\alpha}\right)$,

$$
\sum_{x^{\gamma} f_{j} \in \operatorname{Orb}\left(x^{\delta} f_{i}\right)} M_{x^{\alpha}, x^{\gamma} f_{j}}=\sum_{x^{\gamma} f_{j} \in \operatorname{Orb}\left(x^{\delta} f_{i}\right)} M_{x^{d}, x^{\gamma} f_{j}},
$$

and thus, $\bar{M}_{\operatorname{Orb}\left(x^{\alpha}\right), \operatorname{Orb}\left(x^{\delta} f_{i}\right)}$ is well-defined. We call the matrix $\bar{M}_{F, d, G}$ the orbit matrix. The variable $\bar{y}$ has one entry for every polynomial orbit $\operatorname{Orb}\left(x^{\delta} f_{i}\right)$ denoted $\bar{y}_{\operatorname{Orb}\left(x^{\delta} f_{i}\right)}$. The vector $\bar{b}_{F, d}$ has one entry for every monomial orbit $\operatorname{Orb}\left(x^{\alpha}\right)$, and let $\left(\bar{b}_{F, d}\right)_{O r b\left(x^{\alpha}\right)}=\left(b_{F, d}\right)_{x^{\alpha}}=0$ if $\alpha \neq 0$ and $\left(\bar{b}_{F, d}\right)_{\operatorname{Orb}(1)}=\left(b_{F, d}\right)_{1}=1$. The main result in this section is that, under some assumptions, the system of linear equations $\bar{M}_{F, d, G} \bar{y}=\bar{b}_{F, d, G}$ has a solution if and only if the larger system of linear equations $M_{F, d} y=b_{F, d}$ has a solution.

TheOREM 3.5. Let $F=\left\{f_{1}, \ldots, f_{s}\right\} \subset \mathbb{K}\left[x_{1}, \ldots, x_{n}\right]$, be a polynomial system with $\mathbb{K}$ an algebraically-closed field, and a finite group of permutations $G \subset S_{n}$. Let $M_{F, d}, \bar{M}_{F, d, G}$ denote the matrices defined above. Suppose that the polynomial system $F$ is closed under the action of the group $G$ permuting the indices of variables $x_{1}, \ldots, x_{n}$. Suppose further that the order of the group $|G|$ and the characteristic of the field $\mathbb{K}$ are relatively prime. The degree $d$ Nullstellensatz linear system of equations $M_{F, d} y=b_{F, d}$ has a solution over $\mathbb{K}$ if and only if the system of linear equations $\bar{M}_{F, d, G} \bar{y}=\bar{b}_{F, d, G}$ has a solution over $\mathbb{K}$.

Proof. To simplify notation, let $M=M_{F, d}, b=b_{F, d}$, $\bar{M}=\bar{M}_{F, d, G}$ and $\bar{b}=\bar{b}_{F, d, G}$. First, we show that if the linear system $M y=b$ has a solution, then there exists a symmetric solution $y$ of the linear system $M y=b$ meaning that $y_{x^{\delta} f_{i}}$ is the same for all $x^{\delta} f_{i}$ in the same orbit, i.e., $y_{x^{\gamma} f_{j}}=y_{x^{\delta} f_{i}}$ for all $x^{\gamma} f_{j} \in \operatorname{Orb}\left(x^{\delta} f_{i}\right)$. The converse is also trivially true.

Since the rows and columns of the matrix $M$ are labeled by monomials $x^{\alpha}$ and polynomials $x^{\delta} f_{i}$ respectively, we can think of the group $G$ as acting on the matrix $M$, permuting the entries $M$, i.e., applying $g \in G$ to $M$ gives the permuted matrix $g(M)$ where

$$
g(M)_{g\left(x^{\alpha}\right), g\left(x^{\delta} f_{i}\right)}=M_{x^{\alpha}, x^{\delta} f_{i}} .
$$

Moreover, since $M_{x^{\alpha}, x^{\delta} f_{i}}=M_{g\left(x^{\alpha}\right), g\left(x^{\delta} f_{i}\right)}$ for all $g \in G$, we must have $g(M)=M$, so the matrix $M$ is invariant under the action of the group $G$. Also, since the entries of the variable $y$ are labeled by polynomials of the form $x^{\alpha} f_{i}$, we can also think of the group $G$ as acting on the vector $y$, permuting the entries of the vector $y$, i.e., applying $g \in G$ to $y$ gives the permuted vector $g(y)$ where $g(y)_{g\left(x^{\delta} f_{i}\right)}=y_{x^{\delta} f_{i}}$. Similarly, $G$ acts on the vector $b$, and in particular, $g(b)=b$. Next, we show that if $M y=b$, then $M g(y)=b$ for all $g \in G$ accordingly:

$$
M y=b \Rightarrow g(M y)=g(b) \Rightarrow g(M) g(y)=b \Rightarrow M g(y)=b,
$$

for all $g \in G$. Now, let

$$
y^{\prime}=\frac{1}{|G|} \sum_{g \in G} g(y)
$$

Note we need that $|G|$ is relatively prime to the characteristic of the field $\mathbb{K}$ so that $|G|$ is invertible. Then,

$$
M y^{\prime}=\frac{1}{|G|} \sum_{g \in G} M g(y)=\frac{1}{|G|} \sum_{g \in G} b=b,
$$

so $y^{\prime}$ is a solution. Also, $y_{x^{\delta} f_{i}}^{\prime}=\frac{1}{|G|} \sum_{g \in G} y_{g\left(x^{\delta} f_{i}\right)}$, so $y_{x^{\delta} f_{i}}^{\prime}=y_{x^{\gamma} f_{j}}^{\prime}$ for all $x^{\gamma} f_{j} \in \operatorname{Orb}\left(x^{\delta} f_{i}\right)$. Therefore, $y^{\prime}$ is a symmetric solution as required.

Now, assume that there exists a solution of $M y=b$. By the above argument, we can assume that the solution is symmetric, i.e., $y_{x^{\delta} f_{i}}=y_{x^{\gamma} f_{j}}$ where $g\left(x^{\delta} f_{i}\right)=x^{\gamma} f_{j}$ for some $g \in G$. From this symmetric solution of $M y=b$, we can find a solution of $\bar{M} \bar{y}=\bar{b}$ by setting

$$
\bar{y}_{O r b\left(x^{\delta} f_{i}\right)}=y_{x^{\delta} f_{i}} .
$$

To show this, we check that $(\bar{M} \bar{y})_{O r b\left(x^{\alpha}\right)}=\bar{b}_{O r b\left(x^{\alpha}\right)}$ for every monomial $x^{\alpha}$.

$$
\begin{array}{r}
(\bar{M} \bar{y})_{\operatorname{Orb}\left(x^{\alpha}\right)}=\sum_{\text {all } \operatorname{Orb}\left(x^{\delta} f_{i}\right)} \bar{M}_{\operatorname{Orb}\left(x^{\alpha}\right), \operatorname{Orb}\left(x^{\delta} f_{i}\right)} \bar{y}_{\operatorname{Orb}\left(x^{\delta} f_{i}\right)} \\
=\sum_{\text {all } \operatorname{Orb}\left(x^{\delta} f_{i}\right)}\left(\sum_{x^{\gamma} f_{j} \in \operatorname{Orb}\left(x^{\delta} f_{i}\right)} M_{x^{\alpha}, x^{\gamma} f_{j}}\right) \bar{y}_{O r b\left(x^{\delta} f_{i}\right)} \\
=\sum_{\text {all } \operatorname{Orb}\left(x^{\delta} f_{i}\right)}\left(\sum_{x^{\gamma} f_{j} \in \operatorname{Orb}\left(x^{\delta} f_{i}\right)} M_{x^{\alpha}, x^{\gamma} f_{j}} y_{x^{\gamma} f_{j}}\right) \\
=\sum_{\text {all } x^{\delta} f_{i}} M_{x^{\alpha}, x^{\delta} f_{i}} y_{x^{\delta} f_{i}}=(M y)_{x^{\alpha}} .
\end{array}
$$

Thus, $(\bar{M} \bar{y})_{O r b\left(x^{\alpha}\right)}=\bar{b}_{O r b\left(x^{\alpha}\right)}$ since $(M y)_{x^{\alpha}}=b_{x^{\alpha}}=$ $\bar{b}_{\text {Orb }\left(x^{\alpha}\right)}$.

Next, we establish the converse more easily. Recall that the columns of $\bar{M}$ are labeled by orbits. If there is a solution for $\bar{M} \bar{y}=\bar{b}$, then to recover a solution of $M y=b$, we set

$$
y_{x} \delta f_{i}=\bar{y}_{O r b\left(x^{\delta} f_{i}\right)} .
$$

Note that $y$ is a symmetric solution. Using the same calculation as above, we have that $(M y)_{x^{\alpha}}=(\bar{M} \bar{y})_{O r b\left(x^{\alpha}\right)}$, and thus, $M y=b$.

Example 3.6 (Continuation of Example 3.4). Now consider the action of the symmetry group $G$ generated by the cycle (2,3,4) (a cyclic group of order three). The permutation of variables permutes the monomials and yields a matrix $M_{F, 1, G}$. We have now grouped together monomials and terms within orbit blocks in the matrix below. The blocks will be later replaced by a single entry, shrinking the size of the matrix.

\begin{tabular}{|r||c|c|cc|ccc|ccc|c|ccc|}
\hline & $c_{0}$ & $c_{12}^{1} c_{13}^{1} c_{14}^{1}$ & $c_{12}^{2} c_{13}^{3} c_{14}^{4}$ & $c_{12}^{3} c_{13}^{4} c_{14}^{2}$ & $\cdots$ & $c_{34}^{2} c_{24}^{3} c_{23}^{4}$ \\
\hline \hline$x_{1}^{3}$ & 1 & 1 & 1 & 1 & 0 & 0 & 0 & 0 & 0 & 0 & $\cdot$ & 0 & 0 & 0 \\
\hline$x_{1}^{2} x_{2}$ & 0 & 1 & 0 & 0 & 1 & 0 & 0 & 0 & 0 & 1 & $\cdots$ & 0 & 0 & 0 \\
$x_{1}^{2} x_{3}$ & 0 & 0 & 1 & 0 & 0 & 1 & 0 & 1 & 0 & 0 & $\cdots$ & 0 & 0 & 0 \\
$x_{1}^{2} x_{4}$ & 0 & 0 & 0 & 1 & 0 & 0 & 1 & 0 & 1 & 0 & $\cdots$ & 0 & 0 & 0 \\
\hline$x_{1} x_{2}^{2}$ & 0 & 1 & 0 & 0 & 1 & 0 & 0 & 0 & 0 & 0 & $\cdots$ & 0 & 0 & 0 \\
$x_{1} x_{3}^{2}$ & 0 & 0 & 1 & 0 & 0 & 1 & 0 & 0 & 0 & 0 & $\cdots$ & 0 & 0 & 0 \\
$x_{1} x_{4}^{2}$ & 0 & 0 & 0 & 1 & 0 & 0 & 1 & 0 & 0 & 0 & $\cdots$ & 0 & 0 & 0 \\
\hline$x_{1} x_{2} x_{3}$ & 0 & 0 & 0 & 0 & 0 & 0 & 0 & 1 & 0 & 0 & $\cdots$ & 0 & 0 & 0 \\
$x_{1} x_{2} x_{4}$ & 0 & 0 & 0 & 0 & 0 & 0 & 0 & 0 & 0 & 1 & $\cdots$ & 0 & 0 & 0 \\
$x_{1} x_{3} x_{4}$ & 0 & 0 & 0 & 0 & 0 & 0 & 0 & 0 & 1 & 0 & $\cdots$ & 0 & 0 & 0 \\
\hline$x_{2}^{3}$ & 0 & 0 & 0 & 0 & 1 & 0 & 0 & 0 & 0 & 0 & $\cdots$ & 0 & 0 & 0 \\
$x_{3}^{3}$ & 0 & 0 & 0 & 0 & 0 & 1 & 0 & 0 & 0 & 0 & $\cdots$ & 0 & 0 & 0 \\
$x_{4}^{3}$ & 0 & 0 & 0 & 0 & 0 & 0 & 1 & 0 & 0 & 0 & $\cdots$ & 0 & 0 & 0 \\
\hline$x_{2}^{2} x_{3}$ & 0 & 0 & 0 & 0 & 0 & 0 & 0 & 1 & 0 & 0 & $\cdots$ & 0 & 1 & 0 \\
$x_{3}^{2} x_{4}$ & 0 & 0 & 0 & 0 & 0 & 0 & 0 & 0 & 1 & 0 & $\cdots$ & 0 & 0 & 1 \\
$x_{2} x_{4}^{2}$ & 0 & 0 & 0 & 0 & 0 & 0 & 0 & 0 & 0 & 1 & $\cdots$ & 1 & 0 & 0 \\
\hline$x_{2}^{2} x_{4}$ & 0 & 0 & 0 & 0 & 0 & 0 & 0 & 0 & 0 & 0 & $\cdots$ & 0 & 0 & 1 \\
$x_{2} x_{3}^{2}$ & 0 & 0 & 0 & 0 & 0 & 0 & 0 & 0 & 0 & 0 & $\cdots$ & 1 & 0 & 0 \\
$x_{3} x_{4}^{2}$ & 0 & 0 & 0 & 0 & 0 & 0 & 0 & 0 & 0 & 0 & $\cdots$ & 0 & 1 & 0 \\
\hline$x_{2} x_{3} x_{4}$ & 0 & 0 & 0 & 0 & 0 & 0 & 0 & 0 & 0 & 0 & $\cdots$ & 1 & 1 & 1 \\
\hline
\end{tabular}


The action of the symmetry group generated by the cycle (2,3,4) yields an orbit matrix $\bar{M}_{F, q, G}$ of about a third the size of the original one:

\begin{tabular}{|c|c|c|c|c|c|c|c|c|c|}
\hline & \multicolumn{9}{|c|}{ 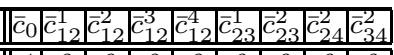 } \\
\hline Orb(1) & 1 & 0 & 0 & 0 & 0 & 0 & 0 & 0 & 0 \\
\hline $\operatorname{Orb}\left(x_{1}^{3}\right)$ & 1 & 3 & 0 & 0 & 0 & 0 & 0 & 0 & 0 \\
\hline $\operatorname{Orb}\left(x_{1}^{2} x_{2}\right)$ & 0 & 1 & 1 & 1 & 1 & 0 & 0 & 0 & 0 \\
\hline $\operatorname{Orb}\left(x_{1} x_{2}^{2}\right)$ & 0 & 1 & 1 & 0 & 0 & 2 & 0 & 0 & 0 \\
\hline $\operatorname{Orb}\left(x_{1} x_{2} x_{3}\right)$ & 0 & 0 & 0 & 1 & 1 & 1 & 0 & 0 & 0 \\
\hline $\operatorname{Orb}\left(x_{2}^{3}\right)$ & 0 & 0 & 1 & 0 & 0 & 0 & 1 & 1 & 0 \\
\hline $\operatorname{Orb}\left(x_{2}^{2} x_{3}\right)$ & 0 & 0 & 0 & 1 & 0 & 0 & 1 & 1 & 1 \\
\hline $\operatorname{Orb}\left(x_{2}^{2} x_{4}\right)$ & 0 & 0 & 0 & 0 & 1 & 0 & 1 & 1 & 1 \\
\hline $\operatorname{Orb}\left(x_{2} x_{3} x_{4}\right)$ & 0 & 0 & 0 & 0 & 0 & 0 & 0 & 0 & 3 \\
\hline
\end{tabular}

\begin{tabular}{|r|||c|c|c|c|c|c|c|c|c|}
\hline & $\bar{c}_{0}$ & $\bar{c}_{12}^{1}$ & $\bar{c}_{12}^{2}$ & $\bar{c}_{12}^{3}$ & $\bar{c}_{12}^{4}$ & $\bar{c}_{23}^{1}$ & $\bar{c}_{23}^{2}$ & $\bar{c}_{24}^{2}$ & $\bar{c}_{34}^{2}$ \\
\hline \hline$O r b(1)$ & 1 & 0 & 0 & 0 & 0 & 0 & 0 & 0 & 0 \\
\hline$O r b\left(x_{1}^{3}\right)$ & 1 & 1 & 0 & 0 & 0 & 0 & 0 & 0 & 0 \\
\hline$O r b\left(x_{1}^{2} x_{2}\right)$ & 0 & 1 & 1 & 1 & 1 & 0 & 0 & 0 & 0 \\
\hline$O r b\left(x_{1} x_{2}^{2}\right)$ & 0 & 1 & 1 & 0 & 0 & 0 & 0 & 0 & 0 \\
\hline$O r b\left(x_{1} x_{2} x_{3}\right)$ & 0 & 0 & 0 & 1 & 1 & 1 & 0 & 0 & 0 \\
\hline$O r b\left(x_{2}^{3}\right)$ & 0 & 0 & 1 & 0 & 0 & 0 & 1 & 1 & 0 \\
\hline$O r b\left(x_{2}^{2} x_{3}\right)$ & 0 & 0 & 0 & 1 & 0 & 0 & 1 & 1 & 1 \\
\hline$O r b\left(x_{2}^{2} x_{4}\right)$ & 0 & 0 & 0 & 0 & 1 & 0 & 1 & 1 & 1 \\
\hline$O r b\left(x_{2} x_{3} x_{4}\right)$ & 0 & 0 & 0 & 0 & 0 & 0 & 0 & 0 & 1 \\
\hline
\end{tabular}

If $|G|$ is not relatively prime to the characteristic of the field $\mathbb{K}$, then it is still true that, if $\bar{M} y=\bar{b}$ has a solution, then $M y=b$ has a solution. Thus, even if $|G|$ is not relatively prime to the characteristic of the field $\mathbb{K}$, we can still prove that the polynomial system $F$ is infeasible by finding a solution of the linear system $\bar{M} y=\bar{b}$.

\subsection{Reducing the Nullstellensatz degree by ap- pending polynomial equations}

We have discovered that by appending certain valid but redundant polynomial equations to the system of polynomial equations described in Lemma 3.1, we have been able to decrease the degree of the Nullstellensatz certificate necessary to prove infeasibility. A valid but redundant polynomial equation is any polynomial equation $g(x)=0$ that is true for all the zeros of the polynomial system $f_{1}(x)=$ $0, \ldots, f_{s}(x)=0$, i.e., $g \in \sqrt{I}$, the radical ideal of $I$, where $I$ is the ideal generated by $f_{1}, \ldots, f_{s}$. In fact, we only really require that $g(x)=0$ holds for at least one of zeros of the polynomial system $f_{1}(x)=0, \ldots, f_{s}(x)=0$ if a zero exists. We refer to a redundant polynomial equation appended to a system of polynomial equations, with the goal of reducing the degree of a Nullstellensatz certificate, as a degree-cutter.

For example, for 3-coloring, consider a triangle described by the vertices $\{x, y, z\}$. Whenever a triangle appears as a subgraph in a graph, the vertices of the triangle must be colored differently. We capture that additional requirement with the equation

$$
x^{2}+y^{2}+z^{2}=0,
$$

which is satisfied if and only if $x \neq y \neq z \neq x$ since $x$, $y$ and $z$ are third roots of unity. Note that the equation $x+y+z=0$ also implies $x \neq y \neq z \neq x$, but we use the equation $x^{2}+y^{2}+z^{2}=0$, which is homogeneous of degree two, because the edge equations from Lemma 3.1 are also homogeneous of degree two, and this helps preserve the balance of monomials in the final certificate.
Consider the Koester graph [9] from Figure 1, a graph with 40 vertices and 80 edges. This graph has chromatic number four, and a corresponding non-3-colorability certificate of degree four. The size (after preprocessing) of the associated linear system required by NulLA to produce this certificate was $8,724,468 \times 10,995,831$ and required 5 hours and 17 minutes of computation time.

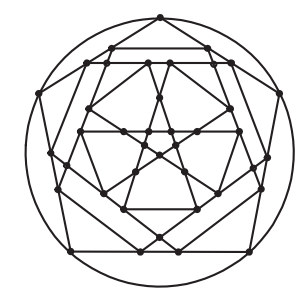

Figure 1: Koester graph

When we inspect the Koester graph in Figure 1, we can see that this graph contains 25 triangles. When we append these additional 25 equations to the system of polynomial equations describing this graph, the degree of the Nullstellensatz certificate drops from four to one, and now, with the addition of the 25 triangle equations, NulLA only needs to solve a $4,626 \times 4,346$ linear system to produce a degree one certificate, which takes 0.2 seconds of computation time. Note that even though we have appended equations to the system of polynomial equations, because the degree of the overall certificate is reduced, the size of the resulting linear system is still much, much less.

These degree-cutter equations for 3-colorability (1) can be extended to $k$-colorability. A $(k-1)$-clique implies that all nodes in the clique have a different color. Then, given the $(k-1)$-clique with the vertices $x_{1}$ through $x_{k-1}$, the equation $x_{1}^{k-1}+x_{2}^{k-1}+\ldots+x_{k-1}^{k-1}=0$ is valid. We conjecture that these equations may also decrease the minimal degree of the Nullstellensatz certificate if one exists.

The degree-cutter equations for 3-colorability (1) are not always sufficient to reduce the degree of the Nullstellensatz. Consider the graph from Figure 2. Using only the polynomials from Lemma 3.1, the graph in Figure 2 has a degree four certificate. The graph contains three triangles: $\{1,2,6\},\{2,5,6\}$ and $\{2,6,7\}$. In this case, after appending the degree-cutter equations for 3-colorability (1) the degree of the minimal Nullstellensatz certificate for this graph is still four.

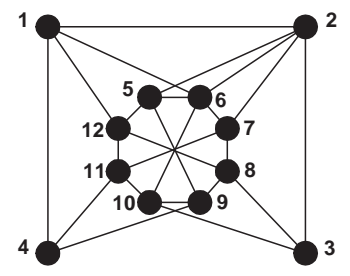

Figure 2: A graph with a degree four certificate.

The difficulty with the degree-cutter approach is in finding candidate degree-cutters and in determining how many of the candidate degree-cutters to append to the system. There is an obvious trade-off here between the time spent finding 
degree-cutters together with the time penalty incurred related to the increased size of the linear system that must be solved versus the benefit of reducing the degree of the Nullstellensatz certificate.

\subsection{Alternative Nullstellensätze}

There is another approach we have found to decrease the minimal degree of the Nullstellensatz certificate. We now introduce the idea of an alternative Nullstellensatz, which follows from the Hilbert Nullstellensatz.

Corollary 3.7 (Alternative Nullstellensatz). $A$ system of polynomial equations $f_{1}(x)=0, \ldots, f_{s}(x)=0$ where $f_{i} \in \mathbb{K}\left[x_{1}, \ldots, x_{n}\right]$ and $\mathbb{K}$ is an algebraically closed field has no solution in $\mathbb{K}^{n}$ if and only if there exist polynomials $\beta_{1}, \ldots, \beta_{s} \in \mathbb{K}\left[x_{1}, \ldots, x_{n}\right]$ and $g \in \mathbb{K}\left[x_{1}, \ldots, x_{n}\right]$ such that $g=\sum \beta_{i} f_{i}$ and the system $f_{1}(x)=0, \ldots, f_{s}(x)=0$ and $g(x)=0$ has no solution.

The Hilbert Nullstellensatz is a special case of this alternative Nullstellensatz where $g(x)=1$. We can easily adapt the NulLA algorithm to use this alternative Nullstellensatz given the polynomial $g$. Here, the polynomial $g$ determines the constant terms of the linear system that we need to solve to find a certificate of infeasibility. The idea here is that the minimal degree of the alternative Nullstellensatz certificate is sometimes smaller than the minimal degree of the ordinary Nullstellensatz certificate.

In the case of 3-colorability (and also more generally $k$ colorability), we may choose $g$ as any non-trivial monomial since $g(x)=0$ implies that $x_{i}=0$ for some $i=1, \ldots, n$, which contradicts that $x_{i}^{3}-1=0$. For the graph in Figure 2, if we choose $g(x)=x_{1} x_{8} x_{9}$ (among others), then the minimal degree of the Nullstellensatz certificate drops to one.

The apparent difficulty in using the alternative Nullstellensatz approach is in choosing $g(x)$. One solution to this problem is to try and find a Nullstellensatz certificate for a set of $g(x)$ including $g(x)=1$. For example, for the graph in Figure 2, we tried to find a certificate of degree one for the set of all possible monomials of degree 3. Since choosing different $g(x)$ only means changing the constant terms of the linear system in NulLA (the other coefficients remain the same), solving for a set of $g(x)$ can be accomplished very efficiently.

\section{EXPERIMENTAL RESULTS}

In this section, we present our experimental results, including a comparison between NulLA and other graph coloring algorithms such as the Alon-Tarsi method [1], the Gröbner basis method, DSATUR and Branch-and-Cut [12]. To summarize, almost all of the graphs tested by NulLA had degree one certificates. This algebraic property, coupled with our ability to compute over $\mathbb{F}_{2}$, allowed us to prove the non3 -colorability of graphs with over a thousand nodes.

\subsection{Methods}

Our computations were performed on machines with dual Opteron nodes, $2 \mathrm{GHz}$ clock speed, and $12 \mathrm{~GB}$ of RAM. No degree-cutter equations or alternative Nullstellensatz certificates were used. We preprocessed the linear systems by removing redundant vertex polynomials via $\left(x_{i}^{3}+1\right)=$ $\left(x_{j}^{3}+1\right)+\left(x_{i}+x_{j}\right)\left(x_{i}^{2}+x_{i} x_{j}+x_{j}^{2}\right)$. Since the graphs that we tested are connected, via the previous equality, we can remove all but one of the vertex polynomial equations by tracing paths from an arbitrarily selected "origin" vertex. We also eliminated unnecessary monomials from the system.

\subsection{Test cases}

We tested the following graphs:

1. DIMACS: The graphs from the DIMACS Computational Challenge $(1993,2002)$ are described in detail at http://mat.gsia.cmu.edu/COLORING02/. This set of graphs is the standard benchmark for graph coloring algorithms. We tested every DIMACS graph whose associated NulLA matrix could be instantiated within 12 GB of RAM. For example, we did not test C4000.5.clq, which has 4,000 vertices and 4,000,268 edges, yielding a degree one NulLA matrix of 758 million non-zero entries and 1 trillion columns.

2. Mycielski: The Mycielski graphs are known for the gap between their clique and chromatic number. The Mycielski graph of order $k$ is a triangle-free graph with chromatic number $k$. The first few instances and the algorithm for their construction can be seen at http: //mathworld.wolfram. com/MycielskiGraph.html.

3. Kneser: The nodes of the Kneser- $(t, r)$ graph are represented by the $r$-subsets of $\{1, \ldots, t\}$. Two nodes are adjacent iff their subsets are disjoint.

4. Random: We tested random graphs in 16 nodes with an edge probability of .27 . This probability was experimentally selected based on the boundary between 3colorable and non-3-colorable graphs and is explained in detail in Section 4.3.

\subsection{Results}

In this section, we present our experimental results on graphs with and without 4 -cliques. We also point out certain properties of NulLA-constructed certificates, and conclude with tests on random graphs. Surprisingly, all but four of the DIMACS, Mycielski and Kneser graphs tested with NulLA have degree one certificates.

The DIMACS graphs are primarily benchmarks for graph $k$-colorability, and thus contain many graphs with large chromatic number. Such graphs often contain 4-cliques. Although testing for graph 3-colorability is well-known to be NP-Complete, there exist many efficient (and even trivial), polynomial-time algorithms for finding 4-cliques in a graph. Thus, we break our computational investigations into two tables: Table 1 contains graphs without 4-cliques, and Table 3 contains graphs with 4-cliques (considered "easy" instances of 3 -colorability). In the tables below, the certificate degree is one, $n$ is the number of vertices and $m$ is the number of edges. For space considerations, we only display representative results for graphs of varying size for each family. The size of the linear systems involved rangef from $15,737 \times 15,681$ up to $45,980,650 \times 46,378,333$ (for the $(8,3)$-Kneser and $(13,5)$-Kneser graphs, respectively).

However, not all of the DIMACS challenge graphs had degree one certificates. We were not able to produce certificates for mug88_1, mug88_25, mug100_1 or mug100_25, even when using degree-cutters and searching for alternative Nullstellensatz certificates. When testing for a degree four certificate, the smallest of these graphs (88 vertices and 146 edges) yielded a linear system with $1,170,902,966$ non-zero 


\begin{tabular}{c|c|c|c} 
Graph & $n$ & $m$ & sec \\
\hline Mycielski 7 & 95 & 755 & .46 \\
Mycielski 9 & 383 & 7,271 & 268.78 \\
Mycielski 10 & 767 & 22,196 & 14835 \\
(8, 3)-Kneser & 56 & 280 & .07 \\
(10,4)-Kneser & 210 & 1,575 & 3.92 \\
(12,5)-Kneser & 792 & 8,316 & 466.47 \\
(13,5)-Kneser & 1,287 & 36,036 & 216105 \\
ash331GPIA & 662 & 4,185 & 13.71 \\
ash608GPIA & 1,216 & 7,844 & 34.65 \\
ash958GPIA & 1,916 & 12,506 & 90.41 \\
1-Insertions_5 & 202 & 1,227 & 1.69 \\
2-Insertions_5 & 597 & 3,936 & 18.23 \\
3-Insertions_5 & 1,406 & 9,695 & 83.45
\end{tabular}

Table 1: Graphs without 4-cliques.

entries and 390,340,149 columns. A matrix of this size is not computationally tractable at this time.

Recall that the certificates returned by NulLA consist of a single vertex polynomial (via preprocessing), and edge polynomials describing either the original graph in its entirety, or a non-3-colorable subgraph from the original graph. For example, if the graph contains a 4-clique as a subgraph, often the Nullstellensatz certificate will only display the edges contained in the 4-clique. In this case, we say that NulLA isolates a non-3-colorable subgraph from the original graph. The size difference between these subgraphs and the input graphs is often dramatic, as shown in Table 2.

\begin{tabular}{c|c|c|c|c} 
Graph & $n$ & $m$ & $\begin{array}{c}\text { subgraph } \\
\text { vertices }\end{array}$ & $\begin{array}{c}\text { subgraph } \\
\text { edges }\end{array}$ \\
\hline miles1500 & 128 & 10,396 & 6 & 10 \\
hamming8-4 & 256 & 20,864 & 19 & 33 \\
Mycielski 10 & 767 & 22,196 & 11 & 20 \\
(12,5)-Kneser & 792 & 8,316 & 53 & 102 \\
dsjc1000.1 & 1,000 & 49,629 & 15 & 24 \\
ash608GPIA & 1,216 & 7,844 & 23 & 44 \\
3-Insertions_5 & 1,406 & 9,695 & 56 & 110 \\
ash958GPIA & 1,916 & 12,506 & 24 & 45
\end{tabular}

Table 2: Input $G$ vs. non-3-colorable subgraph.

An overall analysis of these computational experiments shows that NulLA performs best on sparse graphs. For example, the 3-Insertions_5 graph (with 1,406 nodes and 9,695 edges) runs in 83 seconds, while the 3-FullIns_5 graph (with 2,030 nodes and 33,751 edges) runs in 15027 seconds. Another example is p_hat700-2 (with 700 nodes and 121,728 edges) and will199GPIA (with 701 nodes and 7,065 edges). NulLA proved the non-3-colorability of will199GPIA in 35 seconds, while p_hat700-2 took 30115 seconds.

Finally, as an informal measure of the distribution of degree one certificates, we generated random graphs of 16 nodes with edge probability .27 . We selected this probability because it lies on the boundary between feasible and infeasible instances. In other words, graphs with edge probability less than .27 were almost always 3-colorable, and graphs with edge probability greater than .27 were almost always non-3-colorable. However, we experimentally found that an edge probability of .27 created a distribution that was almost exactly half and half. Of 100 trials, 48 were infeasible. Of those 48 graphs, 40 had degree one certificates and 8 had degree four certificates. Of these remaining 8 instances, we were able to find degree one certificates for all 8 by append- ing degree-cutters or by finding alternative Nullstellensatz certificates. This tentative measure indicates that non-3colorability certificates of degrees greater than one may be rare.

\begin{tabular}{c|c|c|c} 
Graph & $n$ & $m$ & sec \\
\hline miles500 & 128 & 2,340 & 1.35 \\
miles1000 & 128 & 6,432 & 7.52 \\
miles1500 & 128 & 10,396 & 24.23 \\
mulsol.i.5 & 197 & 3,925 & 6 \\
zeroin.i.1 & 211 & 4,100 & 6 \\
queen16_16 & 256 & 12,640 & 106 \\
hamming8-4 & 256 & 20,864 & 621.1 \\
school1_nsh & 352 & 14,612 & 210.74 \\
MANN_a27 & 378 & 70,551 & 9809.22 \\
brock400_4 & 400 & 59,765 & 4548.59 \\
gen400_p0.9_65 & 400 & 71,820 & 9608.85 \\
le450_5d & 450 & 9,757 & 304.84 \\
fpsol2.i.1 & 496 & 11,654 & 93.8 \\
C500.9 & 500 & 112,332 & 72752 \\
homer & 561 & 3,258 & 8 \\
p_hat700-2 & 700 & 121,728 & 30115 \\
will199GPIA & 701 & 7,065 & 35 \\
inithx.i.1 & 864 & 18,707 & 1021.76 \\
qg.order30 & 900 & 26,100 & 13043 \\
wap06a & 947 & 43,571 & 1428 \\
dsjc1000.1 & 1,000 & 49,629 & 2981.91 \\
5-FullIns_4 & 1,085 & 11,395 & 200.09 \\
3-FullIns_5 & 2,030 & 33,751 & 15027.9 \\
& & &
\end{tabular}

Table 3: Graphs with 4-cliques.

\subsection{NulLa vs. other algorithms}

In this section, we compare NulLA to two other algebraic methods for detecting 3-colorability; the Alon-Tarsi (AT) method, and the Gröbner basis (GB) method. We also briefly comment on NulLA's relation to well-known graph coloring heuristics such as DSATUR and Branch-and-Cut [12]. We implemented the Alon-Tarsi method in $\mathrm{C}++$, and used CoCoA Lib [4] to test the Gröbner basis method. For brevity, we do not record any "internal data" about the various algorithmic runs, such as the size of the underlying linear systems solved by NulLA or the maximum number of monomials in the normal forms produced by the Alon-Tarsi method. In the tables below, all certificates have degree one, $n$ refers to the number of vertices, $m$ refers to the number of edges and a "-" signifies that the method was terminated after 4 hours of computation.

The Gröbner basis method refers to simply taking the Gröbner basis of the ideal defined in Lemma 3.1. By Hilbert's Nullstellensatz, the Gröbner basis is a constant if and only if the graph is non-3-colorable.

The Alon-Tarsi method is based on the following (see Section 7 of [1] and references therein):

THEOREM 4.1. Given a graph $G$ with $n$ vertices, let $I_{G}=$ $\left\langle x_{1}^{3}-1, \ldots, x_{n}^{3}-1\right\rangle$. Additionally, let

$$
P_{G}=\prod_{(i, j) \in E(G)}\left(x_{i}-x_{j}\right)
$$

Then $P_{G} \in I_{G}$ if and only if $G$ is non-3-colorable

In order to compute with the Alon-Tarsi method, we note that the set $B=\left\{x_{1}^{3}-1, \ldots, x_{n}^{3}-1\right\}$ is a Gröbner basis for $I_{G}$. Thus, we simply take the normal form of $P_{G}$ with 
respect to $B$. If the normal form is zero, $P_{G} \in I_{G}$, and the graph is non-3-colorable. The efficiency of the Alon-Tarsi method can be increased by incrementally constructing $P_{G}$ [7]: we order the edges, and then find the normal form of $\left(x_{i_{1}}-x_{j_{1}}\right)$ with respect to $B$, and then the normal form of $\left(x_{i_{1}}-x_{j_{1}}\right)\left(x_{i_{2}}-x_{j_{2}}\right)$ with respect to $B$, etc.

We compared NulLA to the Gröbner basis and Alon-Tarsi methods on graphs with and without 4-cliques; results are displayed in Tables 6 and 7, respectively.

NulLA consistently greatly out-performed the Gröbner basis method. For example, on zeroin.i.1, NulLA ran in 6 seconds, while CoCoA Lib took almost one hour. These experimental results indicate that NulLA scales better than the Gröbner basis method.

NulLA also compared extremely favorably with the AlonTarsi method, which usually did not terminate within the requisite time bounds. However, in the special case when the first few vertices and edges of the graph happen to describe a non-3-colorable subgraph (such as a 4-clique, or the Grötzch graph), the Alon-Tarsi method ran very quickly, because of the iterative approach incorporated during implementation. Consider the example of the ninth Mycielski graph (383 vertices and 7,271 edges): the Alon-Tarsi method terminated in .24 seconds, but after we permuted the vertices and edges, the method consumed 9 GB of RAM over 4 hours of computation and only processed 30 edges. This example shows that the Alon-Tarsi method is extremely sensitive to the vertex and edge ordering. If a similiar iterative approach was incorporated either into NulLA or the Gröbner basis method, these algorithms would likewise terminate early in this special case.

As another example of the draw-backs of the Alon-Tarsi method, we considered edge-critical graphs, where the entire input must be read. For example, the odd wheels form a trivial family of edge-critical non-3-color-able graphs. The AlonTarsi method was unable to determine the non-3-colorability of the 17-odd-wheel (18 vertices and 34 edges): after two hours of computation, the normal form contained over 19 million monomials, and had consumed over 8 GB of RAM. The experimental results are displayed in Table 4 .

\begin{tabular}{c|c|c||c||c||c} 
Wheels & $n$ & $m$ & NulLA & GB & $A T$ \\
\hline 9 & 10 & 18 & 0 & 0 & .05 \\
11 & 12 & 22 & 0 & 0 & .74 \\
13 & 14 & 26 & 0 & 0 & 8.47 \\
15 & 16 & 30 & 0 & 0 & 369.45 \\
17 & 18 & 34 & 0 & 0 & - \\
151 & 152 & 302 & .21 & 2.21 & - \\
501 & 502 & 1,002 & 15.58 & 126.83 & - \\
1001 & 1,002 & 2,002 & 622.73 & 1706.69 & - \\
2001 & 2,002 & 4,002 & 12905.6 & - & -
\end{tabular}

Table 4: NulLA, GB and AT on wheel graphs.

We conclude with a short comment about NulLA's relation to DSATUR and Branch-and-Cut [12]. These heuristics return bounds on the chromatic number. In Table 5 (data taken from [12]), we display the bounds returned by Branch-and-Cut (B\&C) and DSATUR, respectively. In the case of these graphs, NulLA determined non-3-colorability very rapidly (establishing a lower bound of four), while the two heuristics returned lower bounds of three and two, respectively. Thus, NulLA returned a tighter lower bound on the chromatic number than B\&C or DSATUR.

\begin{tabular}{ccc||cc||cc||c} 
& \multicolumn{1}{l||}{} & \multicolumn{1}{c||}{ B\&C } & \multicolumn{3}{c|}{ DSATUR } & NulLA \\
\hline Graph & $n$ & $m$ & lb & up & lb & up & sec \\
\hline 4-Insertions_3 & 79 & 156 & 3 & 4 & 2 & 4 & 0 \\
3-Insertions_4 & 281 & 1,046 & 3 & 5 & 2 & 5 & 1 \\
4-Insertions_4 & 475 & 1,795 & 3 & 5 & 2 & 5 & 3 \\
2-Insertions_5 & 597 & 3,936 & 3 & 6 & 2 & 6 & 12 \\
3-Insertions_5 & 1,406 & 9,695 & 3 & 6 & 2 & 6 & 83
\end{tabular}

Table 5: NulLA vs. Branch-and-Cut and DSATUR.

\begin{tabular}{c|c|c||c||c||c} 
Graph & $n$ & $m$ & NulLA & $G B$ & $A T$ \\
\hline miles500 & 128 & 2,340 & 1.35 & 133.91 & .07 \\
miles1000 & 128 & 6,432 & 7.52 & 802.23 & 0 \\
miles1500 & 128 & 10,396 & 24.23 & 2598.84 & .01 \\
mulsol.i.5 & 197 & 3,925 & 6 & 18804.5 & 0 \\
zeroin.i.1 & 211 & 4,100 & 6 & 2753.37 & 0 \\
queen16_16 & 256 & 12,640 & 106 & 59466.9 & 0 \\
hamming8-4 & 256 & 20,864 & 621.1 & - & - \\
le450_5d & 450 & 9,757 & 304.84 & - & - \\
homer & 561 & 3,258 & 8 & - & - \\
dsjc1000.1 & 1,000 & 49,629 & 2981.91 & - & - \\
5-FullIns_4 & 1,085 & 11,395 & 200.09 & - & 557.12 \\
3-FullIns_5 & 2,030 & 33,751 & 15027.9 & - & 3.97
\end{tabular}

Table 6: NulLA, GB, AT on graphs with 4-cliques.

\begin{tabular}{|c|c|c|c|c|c|}
\hline Graph & $n$ & $m$ & NulLA & $G B$ & $A T$ \\
\hline Mycielski 4 & 11 & 20 & 0 & .01 & .22 \\
\hline Mycielski 5 & 23 & 71 & 0 & .08 & .23 \\
\hline Mycielski 6 & 47 & 236 & .04 & 3.99 & .22 \\
\hline Mycielski 7 & 95 & 755 & .46 & 179.94 & .23 \\
\hline Mycielski 8 & 191 & 2,360 & 7.72 & 9015.06 & .23 \\
\hline Mycielski 9 & 383 & 7,271 & 268.78 & - & .22 \\
\hline $\begin{array}{l}\text { Mycielski } 9 \\
\text { permuted }\end{array}$ & 383 & 7,271 & 497.47 & - & - \\
\hline$(6,2)-\mathrm{Kn}$ eser & 15 & 45 & 0 & .03 & 1.87 \\
\hline$(8,3)$-Kneser & 56 & 280 & .07 & 18.39 & - \\
\hline$(10,4)$-Kneser & 210 & 1,575 & 3.92 & 9771.76 & - \\
\hline$(12,5)$-Kneser & 792 & 8,316 & 466.47 & - & - \\
\hline ash331GPIA & 662 & 4,185 & 13.71 & - & - \\
\hline 1-Insertions_4 & 67 & 232 & .04 & 3.71 & - \\
\hline 2-Insertions_4 & 149 & 541 & .26 & 32.42 & - \\
\hline 1-Insertions_5 & 202 & 1,227 & 1.69 & 940.7 & - \\
\hline 3-Insertions_4 & 281 & 1,046 & .97 & 237.69 & - \\
\hline 4-Insertions_4 & 475 & 1,795 & 3.02 & 1596.35 & - \\
\hline 2-Insertions_5 & 597 & 3,936 & 18.23 & - & - \\
\hline
\end{tabular}

Table 7: NulLA, GB, AT on graphs w/o 4-cliques.

\section{CONCLUSION}

We presented a general algebraic method to prove combinatorial infeasibility. We showed that even though the worst-case known Nullstellensatz degree upper bounds are doubly-exponential, in practice for useful combinatorial systems, they are often much smaller and can be used to solve even large problem instances. Our experimental results illustrated that many benchmark non-3-colorable graphs have degree one certificates; indeed, non-3-colorable graphs with certificate degrees larger than one appear to be rare. We also showed that NulLA compares well with other algebraic methods and popular heuristics for colorability.

\section{REFERENCES}

[1] N. Alon, Combinatorial Nullstellensatz, Combinatorics, Probability and Computing, 8, (1999), 7-29. 
[2] D.A. Bayer, "The Division Algorithm and the Hilbert Scheme," Ph.D. Thesis, Harvard University, (1982).

[3] S. Buss and T. Pitassi, Good degree bounds on nullstellensatz refutations of the induction principle, IEEE Conference on Computational Complexity, (1996), 233-242.

[4] CoCoATeam, CoCoA: a system for doing Computations in Commutative Algebra, available at http://cocoa.dima.unige.it.

[5] D. Cox, J. Little and D. O'Shea, "Ideals,Varieties and Algorithms," Springer Undergraduate Texts in Mathematics, Springer-Verlag, New York, (1992).

[6] J.A. De Loera, J. Lee, S. Margulies, S. Onn, Expressing Combinatorial Optimization Problems by Systems of Polynomial Equations and the Nullstellensatz, submitted manuscript 2007, see http://arxiv.org/abs/0706.0578

[7] C.J. Hillar and T. Windfeldt, "An algebraic characterization of uniquely vertex colorable graphs," Journal of Combinatorial Theory Series B, 98 (2008), 400-414.

[8] P. Impagliazzo, P. Pudlák and J. Sgall, Lower bounds for polynomial calculus and the Groebner basis algorithm, Computational Complexity, 8, (1999), 127-144.

[9] E. Koester, On 4-critical planar graphs with high edge density, Discrete Mathematics, 98, (1991), 147-151.

[10] J. Kollar, Sharp effective Nullstellensatz, Journal of the AMS, 1(4), (1988), 963-975.

[11] J.B. Lasserre, Polynomials nonnegative on a grid and discrete optimization, Transactions of the $A M S$, 354(2), (2001), 631-649.

[12] I. Méndez-Díaz and P. Zabala, "A branch-and-cut algorithm for graph coloring," Discrete Applied Mathematics, Vol. 154(5), (2006), 826-847.

[13] M. Laurent, Semidefinite representations for finite varieties, Mathematical Programming, 109, (2007), $1-26$.

[14] L. Lovász, Stable sets and polynomials, Discrete Mathematics, 124, (1994), 137-153.

[15] S. Margulies, "Computer Algebra, Combinatorics, and Complexity: Hilbert's Nullstellensatz and NP-Complete Problems," UC Davis Ph.D. dissertation, in preparation, (2008).

[16] P. Parrilo, Semidefinite programming relaxations for semialgebraic problems," Mathematical Programming, Series B, 96(2), (2003), 293-320. 